\title{
DETERMINING POTENTIAL CAPACITY GAINS WHEN REPURPOSING MV AC CABLES FOR DC POWER TRANSPORTATION
}

\author{
Andrew BURSTEIN \\ TU/e - Netherlands \\ a.w.burstein@tue.nl
}

\author{
Vladimir ĆUK \\ TU/e - Netherlands \\ V.cuk@tue.nl
}

\author{
Erik de JONG \\ DNV GL - Netherlands \\ Erik.deJong@dnvgl.com
}

\begin{abstract}
Due to the changing and growing network, operators are looking at more creative and economic ways to reinforce power transportation paths. One such way, being researched by the DNV GL led FLINK project, is to use existing AC cables as flexible DC-links in the medium voltage level. To determine the capacity gain from switching to DC, one must consider many assumptions. The most influential assumption being the increase in applied voltage magnitude. There is, however, a lack of practical research in MVDC. Some assumptions do not scale directly from higher voltages. For example skin and proximity effects may be negligible. Depending on the assumptions made, the capacity gain can range between $-50 \%$ to $>177 \%$. This paper explores the assumptions used to determine capacity gained by MVDC.
\end{abstract}

\section{INTRODUCTION}

The operation of the distribution network of today is facing challenges that need to be addressed due to the increasing connection of decentralized generation (DG) and new consumer loads (e.g. E-vehicles and heat pumps). The new load trends cause bottlenecks in the network and calls for network reinforcement or restructuring.

One potential solution being explored today by the DNV GL led project, FLINK, is the use of direct current (DC) in the medium voltage (MV) network. The aim being to repurpose existing AC cable systems that experience congestion with a flexible DC-link to gain capacity and provide ancillary services through active control systems.

The idea to repurpose an AC cable for DC has become a more popular area of exploration lately. For example, two current projects in the UK: Angle-DC in MV [1] and Top and Tail in LV [2], [3]. However, the area of MVDC research is still in an early stage and lacks practical experience. Because of this shortage of knowledge, HVDC transmission research (a relatively well-developed field) is often used and scaled down. One must be careful in this process as not all phenomena from HVDC scale down directly, e.g. skin and proximity effects.

One of the major goals of switching operation of a cable from AC to DC is to unlock capacity from existing circuits. As will be shown in this paper, an overall capacity gain by switching to DC is not always guaranteed. Depending on the topology and assumptions made, the capacity 'gain', can be anywhere from $-50 \%$ (asymmetric monopole with conductive return) to over $177 \%$ (3 parallel symmetric monopoles including $\mathrm{N}-1$ contingency).
This paper will go through, the different areas in which capacity can be gained by switching to DC and carefully analyze how and when these gain/loss factors are applicable.

\section{BACKGROUND}

The power transferred through a DC-link is purely active. For AC, active power can be shown as:

Where:

$$
P=m \cdot U_{0} \cdot I_{0} \cdot \cos \varphi
$$

$$
\begin{array}{cl}
m & =\text { Number of balanced AC phases } \\
U_{0} & =\text { Nominal AC phase voltage } \\
I_{0} & =\text { Nominal AC phase current } \\
\cos \varphi & =\text { Displacement power factor }
\end{array}
$$

For a balanced AC system, the value of active power per phase is multiplied by the number of phases because no return path is required. For DC, however, a return path is required and the amount of active power is based on the DC voltage (line-to-line) and number of circuits:

$$
P^{\prime}=m^{\prime} \cdot U^{\prime} \cdot I^{\prime}
$$

Where:

$$
\begin{aligned}
m^{\prime} & =\text { Number of DC circuits } \\
U^{\prime} & =\text { DC circuit voltage } \\
I^{\prime} & =\text { DC current }
\end{aligned}
$$

Combining (1) and (2), it can be seen that there are four areas that can affect the capacity gain/loss: the number of conductors, voltage, current, and power factor. Seen as:

$$
\frac{P^{\prime}}{P}=\frac{m^{\prime}}{m} \cdot \frac{U^{\prime}}{U_{0}} \cdot \frac{I^{\prime}}{I_{0}} \cdot \frac{1}{\cos \varphi}
$$

There is always a capacity gain from the change to unity power factor, so this factor will not be looked into further. For the first factor, the number of loaded conductors is determined by the link topology.

\section{DC-LINK TOPOLOGY}

The FLINK flexible DC-link will utilize voltage source converters (VSCs) as these offer the most controllability and ancillary services for the network. Furthermore, they do not require a reversal of voltage polarity to reverse power flow (especially important for XLPE cables), and the DC-voltage can be controlled for a set magnitude. 
The link configurations for VSCs can be classified into symmetric or asymmetric. The symmetric topologies have equal positive and negative voltages and include: monopolar and bipolar (with grounded or conductive neutral) configurations. The asymmetric topologies are: monopolar with a ground or conductive return. These can be found graphically depicted in [4], and the connections with voltages can be found at the end of this paper in Table 2 (note that $U^{\prime}$ is not equivalent for each configuration). Because of the locations of most MV applications, it is assumed that continuous ground return currents will not be permitted; however, ground return may be able to be used for temporary conditions, e.g. reconfiguration time after a converter failing for a symmetric bipole with grounded neutral.

What is most important to note from these configurations is that, because the voltage is limited to the cable's maximum phase voltage, from the link's perspective, the symmetric monopole and bipole-with-grounded-neutral-point configurations are identical, as seen in Table 2. When choosing between using a symmetric monopole or bipole, other factors must therefore be considered; one being the amount of reliability desired. This will be covered in more detail in the section: $N-1$ contingency.

For asymmetrical configurations, the DC voltage is half the magnitude of the symmetrical cases because the return path is at ground potential. For the case of the asymmetric monopole with conductive return, this leads to a reduced transfer capacity with the same number of components as the symmetrical topologies with grounded neutral points.

For this reason, and that continuous ground return is not permissible, from this point on, asymmetric topologies for normal operation will be disregarded.

If we now assume that each phase can handle the peak AC phase voltage continuously as the DC voltage (as often used [1], [3], [5]), then the effect of the topology alone can be seen on the capacity gains in Table 2 (neglecting the current capacity factors which will be discussed in the following sections). For the case of a single 3-core cable, using the peak AC phase voltage can exceed the magnitude of the peak line-to-line voltage, and is therefore limited by this value. It is important to also note that there is only a significant capacity gain if two AC cables are available.

\section{CABLE LIMITATIONS}

When determining the capacity gain of an AC cable for DC use, the above assumptions can be used for an estimation. However, these are conservative assumptions and may not fully consider the changes occurring to the system and insulator properties by switching to DC.

Two major categories for cable ageing and failure are thermal and electrical. Higher thermal loading causes accelerated ageing via thermo-chemical degradation. Also, if heat cannot escape the cable fast enough, it can lead to thermal breakdown. Higher electric field strengths can increase partial discharge (PD) activity and accelerate electric tree growth, leading to failure.

The impact of changing the voltage and current characteristics on these attributes will be explored below.

\section{DC voltage limitation}

\section{Electric stress inversion}

One concern for switching from AC to DC is the inversion of the electric field as graphically depicted in Figure 1. The major concern being that the outside of the insulation may experience more damage and deformations over time (e.g. expansion and retraction due to loading cycles) leading to raised concern for the stress level in this area.

The change in stress distribution is caused by the insulation going from purely capacitive to purely resistive operation.

Because all of the variables to calculate the DC electric stress distribution rely upon each other (e.g. conductivity as a function of temperature and electric field; temperature as a function of electric field and conductivity; etc.), accurately determining the electric field becomes complicated even for the simplest geometries [6]-[8]. Without relying on multi-physical simulations, a commonly used estimation for the electric field distribution is [9]-[11]:

$$
\begin{gathered}
E(r)=\frac{d U_{\phi}}{d r}=\frac{\delta U_{\phi}\left(r / r_{i}\right)^{\delta-1}}{r_{i}\left[1-\left(r_{c} / r_{i}\right)^{\delta}\right]} \\
\delta=\frac{\frac{\alpha \Delta \theta}{\ln \left(r_{i} / r_{c}\right)}+\frac{\beta U_{\phi}}{r_{i}-r_{c}}}{\frac{\beta U_{\phi}}{r_{i}-r_{c}}+1}
\end{gathered}
$$

Where: $\alpha$ is the temperature coefficient of electrical resistivity of the insulator $\left[\left({ }^{\circ} \mathrm{C}\right)^{-1}\right] ; \beta$ is the electrical stress coefficient of the insulator $[\mathrm{m} / \mathrm{V}]$; and the other variables are defined in Figure 1.

Using (4) and cable properties from [9], for paper insulated AC cables with a DC voltage, Table 1 gives the stress magnitudes: at the conductor under no-load conditions (which gives the same curve as would be found for an AC voltage of equivalent RMS magnitude); and at the insulator-screen interface for no-load and fully-loaded conditions.

There are a few important differences to note between HV and MV. The first is the general order of magnitude of the stresses. Naturally, for the lower voltage, the stress is also much lower; so, many concerns for electric breakdowns from the HV level do not scale down to MV. For the concern of the electric field at the insulator edge, in $\mathrm{HV}$ the 


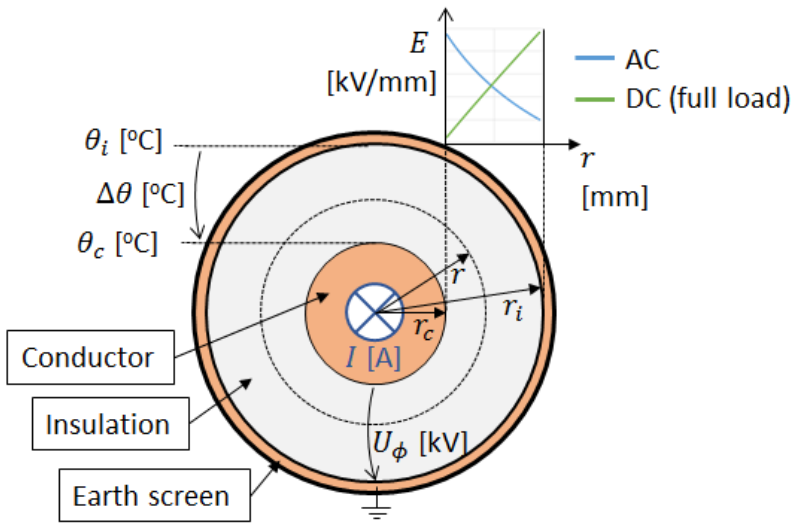

Figure 1 Cable cross-section for electric field calculations change between no-load and full-load is over $5.5 \mathrm{kV} / \mathrm{mm}$, whereas for $\mathrm{MV}$, the change is less than $0.5 \mathrm{kV} / \mathrm{mm}$. This lower change also results in less concern for the inversion.

\section{Breakdown strength}

Electrical breakdown can occur if a conductive path is created between the conductor and ground. This path can be formed by PDs carbonizing the material it contacts and creating an electric tree. PDs occur, among other reasons, due to high electrical stresses and defects in insulation. The permissible electric field strength for DC, however, is higher than it is for AC [11]-[13].

One PD, of course, does not lead to a breakdown, but by the path formed by many PDs. In the case of AC, once the inception voltage has been breached, PDs occur at least twice per cycle. For DC, on the other hand, because there is no $d V / d t$, the charge across a void is dependent on leakage current, yielding a much slower recovery time. There is a give and take, however: since the breakdown strength for DC is higher, if a PD occurs, it will have greater strength leading to more damage per PD.

It is important to note, that for paper cables, PDs are much less of a problem than for XLPE. This is due to the selfhealing properties of oil-impregnated paper. For XLPE, however, once a PD is detected, if no action is taken, it is likely that total failure will occur in a short time (minutes to days for AC) according to cable experts from DNV GL.

Earlier in this paper, the peak AC voltage was assumed a reasonable DC voltage to apply to an aged AC cable; however, this value can be much higher, e.g. over twice the rated RMS voltage [13]. Furthermore, AC cables' voltage is rated with the knowledge that it will need to be able to Table 1 Electric stress calculation results with DC voltage

\begin{tabular}{|c|l|c|c|}
\hline \multicolumn{2}{|c|}{ Voltage level } & MV & HV \\
\hline \multicolumn{2}{|c|}{$\boldsymbol{U}_{\boldsymbol{d} \boldsymbol{c}, \boldsymbol{\phi}}[\mathbf{k V}]$} & 8.2 & 367 \\
\hline \multicolumn{2}{|c|}{$\mathbf{A}\left[\mathbf{m m}^{\mathbf{2}}\right]$} & 400 & 1600 \\
\hline $\boldsymbol{E}\left(\boldsymbol{r}_{\boldsymbol{c}}\right)[\mathbf{k V} / \mathbf{m m}]$ & $\boldsymbol{I}=\mathbf{0}$ & 4.8 & 23.5 \\
\hline \multirow{2}{*}{$\boldsymbol{E}\left(\boldsymbol{r}_{\boldsymbol{i}}\right)[\mathbf{k V} / \mathbf{m m}]$} & $\boldsymbol{I}=\mathbf{0}$ & 4.2 & 16.0 \\
\cline { 2 - 4 } & $\boldsymbol{I}=\boldsymbol{I}_{\text {nom }}$ & 4.7 & 21.5 \\
\hline
\end{tabular}

withstand certain over-voltages on a regular basis. Therefore, for AC there is an insulation headroom applied to each cable of at least $2.5 U_{0}$ [14]. For DC, because the voltage is more controlled, this amount of headroom may be decreased to $1.7 U_{\phi}^{\prime}[14]$, allowing for at least $47 \%$ more voltage capacity, seen in Table 2 as headroom factor, $h$.

The new voltage rating is very important, because, as will be shown later in this paper, the dominant source of capacity gain when switching from $A C$ to $D C$ is from the increase in applied voltage. For this reason, as there is not much research yet on MVAC cables operating with DC and its effects on its health and operation, further, experimentally based research must be performed to determine the true, maximum, safe voltage that can be applied. The FLINK project is planning to perform lab tests to determine this value and other potential challenges by studying cables' PD activity and breakdown strengths. It is anticipated that these results will be able to further be used to develop an online PD-measuring system and active DCvoltage controller to optimize the operation and lifetime of the cable system.

\section{Thermal breakdown}

Thermal breakdown can occur if the electric field becomes high enough that the dielectric losses generate enough heat that the total heat of the cable cannot be dissipated as fast as it is created. However, for MVDC, the dielectric losses are negligible [15], and it is expected that electrical breakdown from defects will occur well before a thermal breakdown voltage is reached, especially due to the lower quality of insulation for MV cables compared to HV cables.

\section{Current limitation}

The load current of any cable is limited by the thermal properties of the insulation. The temperature of the insulation is affected largely by the conductor losses, but is also dependent on: thermal resistances of the components and surroundings; adjacent heat sources; dielectric losses; and losses due to induced currents in metallic layers. This can be seen in the formula used to calculate the nominal AC current in the European standard IEC 60287 [15]. Assuming a 3-phase cable or both ends bonded single-core cables, the armor and shield losses can be neglected [15]. For MV $\mathrm{DC}$, the dielectric losses, $W_{d}$, (being caused mainly by dipole losses) can also be neglected.

From [15], the following relationship of DC to AC current capacity can be found:

$$
\frac{I^{\prime}}{I_{0}}=\sqrt{\left(\frac{R}{R^{\prime}}\right)\left[\frac{T_{1}+n T_{234}}{T_{1}+n^{\prime} T_{234}}\right]\left\{\frac{\Delta \theta}{\Delta \theta-W_{d}\left[0.5 T_{1}+n T_{234}\right]}\right\}}
$$

Where:

$R=$ Resistance per unit length of the conductor at max. operating temperature $[\Omega / \mathrm{m}]$ 
$n=$ Number of load-carrying conductors

$T_{1} \quad=$ Thermal resistance per unit length between one conductor and the sheath $[(\mathrm{K} \cdot \mathrm{m}) / \mathrm{W}]$

$T_{234}=$ Sum of the thermal resistances per unit length of the bedding between sheath and armor, external serving, and surrounding medium $[(\mathrm{K} \cdot \mathrm{m}) / \mathrm{W}]$

A prime is used to indicate the case of DC.

In (6), three distinct factors can be identified. From left-toright, these factors will be further referred to as: the skin and proximity effect factor $\left(k_{S P}\right)$; thermal interaction factor $\left(k_{\tau}\right)$; and dielectric loss factor $\left(k_{D}\right)$. Where each factor includes the square root, making:

$$
I^{\prime}=\left(k_{S P} \cdot k_{\tau} \cdot k_{D}\right) I_{0}
$$

\section{Skin and proximity effect factor $\left(\boldsymbol{k}_{S P}\right)$}

In AC, the effective current carrying area of the conductor may be decreased due induced currents in the conductor from oscillating currents pushing the load current into smaller areas of the conductor (from its own current - skin effect; from adjacent currents - proximity effect), reducing its transport capacity. For DC, these effects do not occur.

The loss of these effects can sometimes be found cited as a significant reason to switch to DC [3], [16]. However, this is not always the case. Using the formulas from [15], the resistive enhancements $\left(R / R^{\prime}\right)$ are calculated with maximum conductor temperature of $70^{\circ} \mathrm{C}$. (Note that for current capacity, because it is based on the conductive losses, the capacity gain is the square root of the resistive enhancement.) As seen in Figure 2, as the conductor size increases, the current capacity greatly increases. However, in the Dutch MV distribution network, the conductor size is at most $630 \mathrm{~mm}^{2}$, and very often lower. The conductors used are also often aluminum; in which case means that the maximum current enhancement will be a little over $2 \%$.

\section{Thermal interaction factor $\left(\boldsymbol{k}_{\tau}\right)$}

As mentioned before, the current is largely limited by the thermal restraints of the insulator. If there are other heat sources in the vicinity (e.g. a 3-core cable), the current of one conductor is decreased to handle the extra thermal loading.

When operating a 3-core cable with DC, it is possible to have one conductor that is normally not loaded. (Seen in (6) as $n^{\prime}=2$.) Using the thermal resistances calculated with values from [9] and formulas from [15], $k_{\tau}$ ranges from 12 to $11 \%$ for cross-sectional areas from 150 to 630 $\mathrm{mm}^{2}$ respectively, with an uncertainty of $+4 \% /-6 \%$ (depending on the value of the geometric factor).

While this enhancement can be quite significant, it does not make up for the loss of a conducting path. Being able to use all conductors at a lower current (e.g. with two 3-

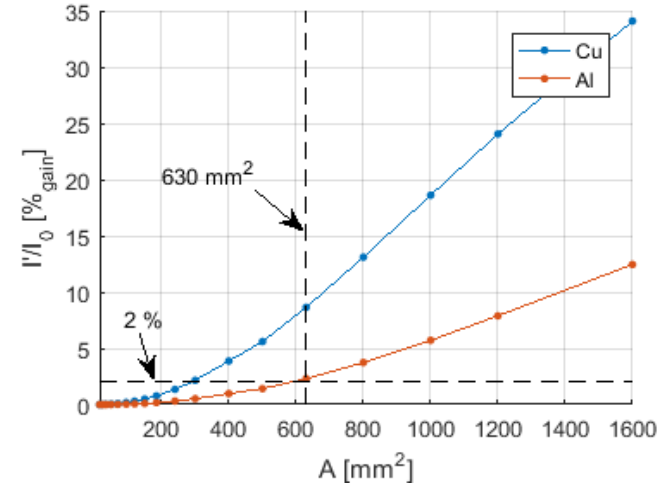

Figure 2 Current enhancement due to skin and proximity effect core cables) allows for greater power transfer capacity.

\section{Dielectric loss factor $\left(k_{D}\right)$}

Heat generated within the dielectric is mainly due to friction losses from dipoles rotating with the voltage frequency. By switching to DC these losses become null leaving only the much smaller leakage current losses. For HV applications this may lead to an increase in current capacity, however, for MV cables, because the capacitance is much smaller, the AC dielectric losses can already be considered negligible. For example, for the paper-insulated $\mathrm{MV}$ cable from the previous section, $k_{D}$ was found to be $0.16 \%$. (This drops to less than $0.03 \%$ for an XLPE cable of the same cross-sectional area.)

\section{N-1 CONTINGENCY}

Until now, we have only considered the cable's nominal rated current, however, for $\mathrm{N}-1$, there must be significant excess capacity and equipment to take over a loss of a component without a loss of load. For the case of one AC cable, we assume there is no $\mathrm{N}-1$ contingency and the enhancement does not change. For the two cable case, however, if $\mathrm{N}-1$ is considered, then each cable can be normally loaded up to half its nominal current. For DC in this case, a symmetric monopole with one converter per station is not possible (i.e. if one converter fails the entire link is down). A bipolar connection, on the other hand, could run temporarily as an asymmetric monopole (with ground or conductive return) if one converter fails. During this recovery period, the phase voltage is reduced by half, the load current is doubled, but the number of circuits remains the same; therefore the enhancement given in Table 2 is not affected.

If a symmetric monopole is made per two conductors (i.e. three converters per station), then if one converter were to fail, the other two circuits could take the load. In this case, the enhancement between the N-1 AC and N-1 DC becomes one-third greater than the values given in Table 2 .

\section{CONCLUSIONS}

When determining the capacity gain possible by switching from AC to DC, generally, the peak AC voltage is considered as a safe DC phase voltage, and the capacity gain is 
Table 2 Capacity gain overview (no N-1) (conductors: green - current carrying; blue - connected neutral; red $X$ - not connected)

\begin{tabular}{|c|c|c|c|c|c|c|c|c|c|c|c|c|}
\hline \multirow{3}{*}{$\begin{array}{c}\text { Link } \\
\text { topology }\end{array}$} & \multicolumn{6}{|c|}{1 AC cable } & \multicolumn{6}{|c|}{2 AC cables } \\
\hline & \multirow{2}{*}{ Cable } & \multirow{2}{*}{$\frac{m^{\prime}}{m}$} & \multirow{2}{*}{$\frac{U^{\prime}}{U_{0}}$} & \multirow{2}{*}{$\frac{I^{\prime}}{I_{0}}$} & \multicolumn{2}{|c|}{$\boldsymbol{P}^{\prime} / \boldsymbol{P}_{0}$} & \multirow{2}{*}{ Cable } & \multirow{2}{*}{$\frac{m^{\prime}}{m}$} & \multirow{2}{*}{$\frac{U^{\prime}}{U_{0}}$} & \multirow{2}{*}{$\frac{I^{\prime}}{I_{0}}$} & \multicolumn{2}{|c|}{$P^{\prime} / P_{0}$} \\
\hline & & & & & No $h$ & $\mathbf{w} / \boldsymbol{h}$ & & & & & No $h$ & w/h \\
\hline $\begin{array}{c}\text { Symmetric } \\
\text { monopole }\end{array}$ & & & & & & & & 1 & $2 \sqrt{2}$ & $k$ & 1.41 & 2. 08 \\
\hline $\begin{array}{c}\text { Bipolar } \\
\text { (gnd neutral) }\end{array}$ & & 1 & $\sqrt{6}$ & $k_{S P}$ & $\begin{array}{l}0.82 \\
\cdot k_{S P}\end{array}$ & $\begin{array}{l}1.20 \\
\cdot k_{\mathrm{SP}}\end{array}$ & & $\overline{2}$ & $2 \mathrm{~V} 2$ & $\kappa_{S P}$ & $\cdot \boldsymbol{k}_{S P}$ & $\cdot \boldsymbol{k}_{S P}$ \\
\hline $\begin{array}{c}\text { Bipolar } \\
\text { (cond. neutral) }\end{array}$ & & 3 & & $\cdot k_{\tau}$ & $\cdot \boldsymbol{k}_{\tau}$ & $\boldsymbol{k}_{\tau}$ & & $\frac{1}{3}$ & $2 \sqrt{2}$ & $\begin{array}{l}k_{S P} \\
\cdot k_{\tau}\end{array}$ & $\begin{array}{l}0.94 \\
\cdot \boldsymbol{k}_{S P} \\
\cdot \boldsymbol{k}_{\tau}\end{array}$ & $\begin{array}{l}1.39 \\
\cdot \boldsymbol{k}_{S P} \\
\cdot \boldsymbol{k}_{\tau}\end{array}$ \\
\hline $\begin{array}{l}\text { Asymmetric } \\
\text { monopole } \\
\text { (cond. return) }\end{array}$ & & $\frac{1}{3}$ & $\sqrt{2}$ & $\begin{array}{l}k_{S P} \\
\cdot k_{\tau}\end{array}$ & $\begin{array}{l}0.47 \\
\cdot \boldsymbol{k}_{S P} \\
\cdot \boldsymbol{k}_{\tau}\end{array}$ & $\begin{array}{l}0.69 \\
\cdot k_{S P} \\
\cdot k_{\tau}\end{array}$ & & $\frac{1}{2}$ & $\sqrt{2}$ & $k_{S P}$ & $\begin{array}{l}0.71 \\
\cdot k_{S P}\end{array}$ & $\begin{array}{l}1.04 \\
\cdot k_{S P}\end{array}$ \\
\hline $\begin{array}{l}\text { Asymmetric } \\
\text { monopole } \\
\text { (gnd return) }\end{array}$ & & 1 & $\sqrt{2}$ & $k_{S P}$ & $\begin{array}{c}1.41 \\
\cdot k_{S P}\end{array}$ & $\begin{array}{l}2.08 \\
\cdot k_{S P}\end{array}$ & & 1 & $\sqrt{2}$ & $k_{S P}$ & $\begin{array}{l}1.41 \\
\cdot k_{S P}\end{array}$ & $\begin{array}{l}2.08 \\
\cdot k_{S P}\end{array}$ \\
\hline
\end{tabular}

calculated with this magnitude and the topology chosen. Other factors are said to contribute to capacity gain, e.g. skin and proximity effects, however, for the MV level, these losses are largely negligible. For the greatest capacity gain, one must have at least two AC cables available utilizing all conductors for power transfer. With this, the current capacity is not significantly changed, leaving the full capacity gain equal to the increased voltage rating.

Because of this heavy reliance on voltage, and a lack of research in this area, further studies are planned by FLINK to determine how high this value can be safely set.

This research has looked primarily at cables, however, another important aspect is to determine how other parts of the cable system will be affected, i.e. joints and terminations. These will also be explored in the future by FLINK.

\section{Acknowledgments}

The FLINK project is funded by the Rijksdienst voor Ondernemend Nederland ( $\mathrm{RvO}$ ) in the framework of Topconsortium voor Kennis en Innovatie (TKI) Urban Energy and coordinated by DNV·GL. This project also benefits from a consortium of experts from SMEs: Early Minute and EMForce; and DNOs: Alliander and Stedin.

\section{REFERENCES}

[1] 2015, “Angle-DC: 2015 Electricity network Innovation Competition.”

[2] Y. Liu, X. Cao, and M. Fu, 2015, “The Upgrading Renovation of an Existing XLPE Cable Circuit by Conversion of AC Line to DC Operation," IEEE Trans. Power Deliv., no. 99, pp. 1-1.

[3] D. Antoniou, A. Tzimas, and S. M. Rowland, 2013, "DC utilization of existing LVAC distribution cables," IEEE Electrical Insulation Conference, pp. 518-522.
[4] 2012, "HVDC Light ${ }^{\circledR}$ It's time to connect.” ABB, p. 14.

[5] D. Nilsson and A. Sannino, 2004, "Efficiency analysis of low- and medium-voltage dc distribution systems," IEEE Power Engineering Society General Meeting, 2004., vol. 2, pp. 23162322.

[6] J. J. O’Dwyer, 1969, “Theory of Dielectric Breakdown in Solids,” J. Electrochem. Soc., vol. 116, no. 2, p. 239.

[7] M. Jeroense, 1997, "Charges and Discharges in HVDC Cables-in particular in mass-impregnated HVDC cables,” Delft University of Technology.

[8] C. C. Reddy and T. S. Ramu, 2007, "Estimation of thermal breakdown voltage of HVDC cables - A theoretical framework," IEEE Trans. Dielectr. Electr. Insul., vol. 14, no. 2, pp. 400-408.

[9] G. Moore, Ed., 1997, Electric Cables Handbook, 3rd ed. BICC Cables Ltd.

[10] C. K. Eòll, 1975, "Theory of Stress Distribution in Insulation of High- Voltage DC Cables: Part I and II,” IEEE Trans. Electr. Insul., vol. EI-10, no. 1, pp. 27-54.

[11] F. H. Kreuger, 1995, Industrial High DC Voltage. Delft University Press: Delft.

[12] E. W. Kimbark, 1971, Direct Current Transmission. John Wiley \& Sons, Inc.

[13] F. Lesur et al., 2015, "Upgrading \& Uprating of Existing Cable Systems.”

[14] J. Arrillaga, N. Watson, and Y. Liu, 2007, Flexible power transmission.

[15] 2006, "International Standard IEC 60287-1-1: Electric cables - Calculation of the current rating.”

[16] S. Hay, C. Cleary, G. Mcfadzean, J. Mcgray, and N. Kelly, 2015, "MVDC Technology Study Market Opportunities and Economic Impact.” 